Document downloaded from:

http://hdl.handle.net/10251/142159

This paper must be cited as:

Gallardo Bermell, S.; Ródenas Diago, J.; Verdú Martín, GJ. (02-2). Unfolding X-ray spectra using a flat panel detector. Determination of the accuracy of the method with the Monte Carlo method. Radiation Physics and Chemistry. 155:233-238.

https://doi.org/10.1016/j.radphyschem.2018.09.014

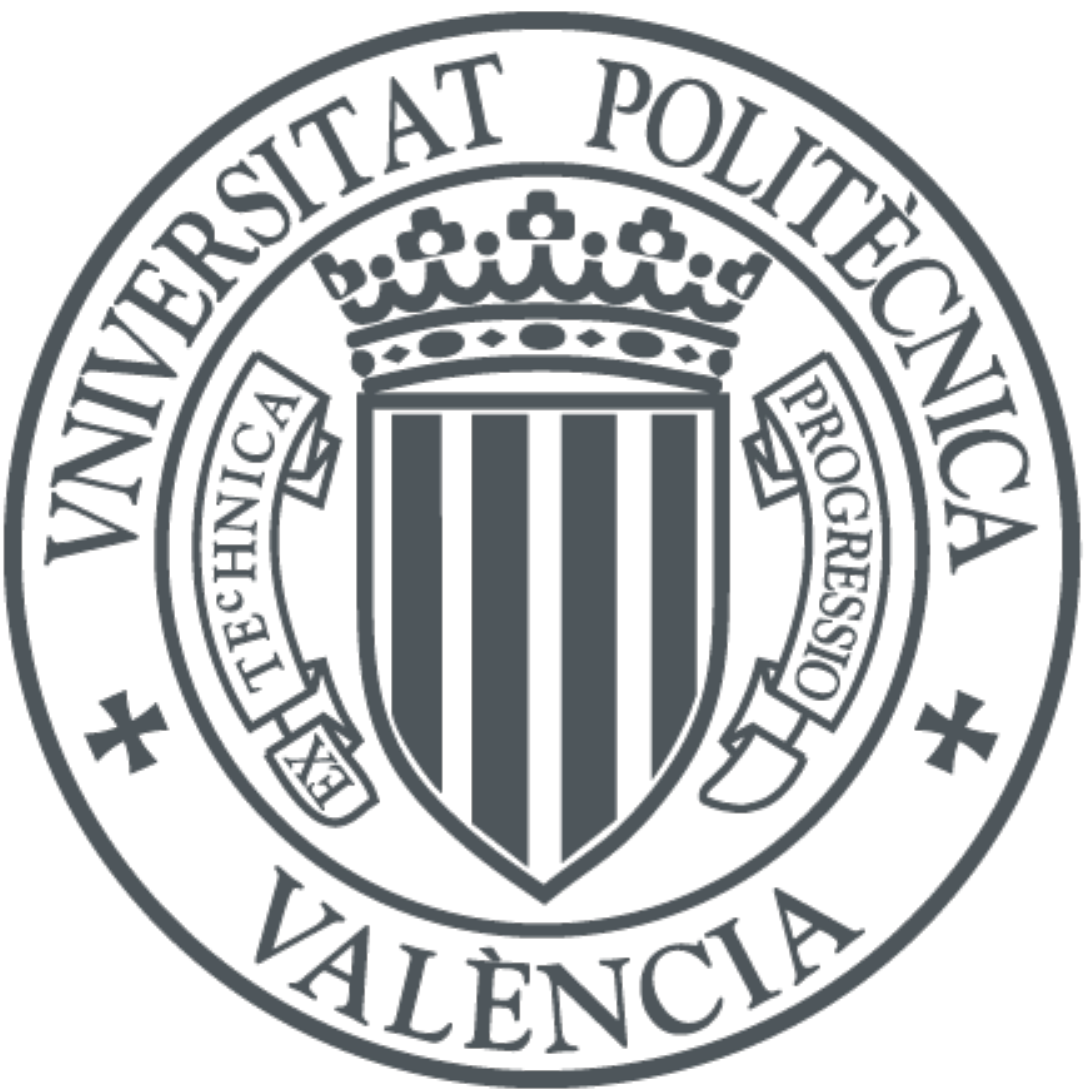

The final publication is available at

https://doi.org/10.1016/j.radphyschem.2018.09.014

Copyright Elsevier

Additional Information 


\title{
UNFOLDING X-RAY SPECTRA USING A FLAT PANEL DETECTOR. DETERMINATION OF THE ACCURACY OF THE METHOD WITH THE MONTE CARLO METHOD
}

\author{
S. Gallardo, J. Ródenas and G. Verdú \\ Instituto Universitario de Seguridad Industrial, Radiofísica y Medioambiental. \\ Universitat Politècnica de València, València, Spain
}

\begin{abstract}
The primary X-ray spectrum depends on different parameters such as high voltage, filament current, high voltage ripple, anode angle and thickness of filter material. The objective of this work is to determine whether the unfolding technique based on the Tikhonov regularization method is accurate enough to estimate the X-ray spectrum when slight changes in the operation variables are considered. In this frame, several X-ray spectra are considered (extracted from the IPEM78 Catalogue Report) varying the main operation variables of the $X$-ray tube (high voltage, voltage ripple, filter thickness and filter material). With those spectra, the corresponding absorbed dose curves are obtained by simulation with a MCNP5 model reproducing a flat panel detector and a PMMA wedge. Once the absorbed dose curves are simulated and applying the unfolding Tikhonov regularization method, the unfolded spectrum is obtained, which is finally compared with the theoretical one (IPEM78 Catalogue Report). Discrepancies between unfolded and primary X-ray spectra can be attributed to the fact that this is an ill-posed problem, and the unfolding of the spectrum is strongly affected by the method used to improve the conditioning of the response function (response matrix).
\end{abstract}

\section{Introduction}

Accurate experimental measurements of X-ray spectra in the radiodiagnostic energy range present important difficulties due to the high fluence rate of photons. Experimentally, the X-ray spectrum can be estimated by measuring scattered radiation or using attenuating materials. Anyway, it is difficult to determine the X-ray spectrum with acceptable accuracy because measurements are affected by several uncertainties. Furthermore, pulse height distributions or spectra measured needs to be unfolded to estimate the primary X-ray spectrum.

In this frame, the unfolding method used to manage the measured data is normally the key to achieve acceptable results in terms of accuracy. Different methods can be found in literature for spectrum unfolding. For instance, the Maximum Entropy Method (MEM) has been successfully applied for photon and neutron spectrometry unfolding (Los Arcos, 1996). MAXED and GRAVEL computer codes contained in the UMG (Unfolding with MAXED and GRAVEL) developed by the Physikalisch Technische Bundesanstalt (Reginatto, 2003) have been widely tested. The MAXED computer code has been applied for neutron unfolding using scintillation detector measurements of photon calibration spectra, and of multisphere neutron spectrometer measurements of cosmic-ray (Reginatto et al., 2002). MAXED and GRAVEL codes have been also used for unfolding data from Bonner spheres in experiments to drive a sub-critically reactor with a Linear Electron Accelerator (LINAC). Artificial Neural Networks and Genetically Evolved Artificial Neural Networks (Ortiz-Rodriguez et al., 2011) have been tested with the aim of unfolding neutron spectra from count rates of a Bonner sphere spectrometer. In the field of radiodiagnostic measurements, Reginatto and Zimbal (2008) have applied Bayesian and Maximum Entropy methods to measurements performed with neutron spectrometers. Fernandez et al. (2014) developed the code UMESTRAT (Unfolding Maximum Entropy STRATegy), which applies a semi-automatic strategy to solve the unfolding problem by using a suitable combination of MAXED and GRAVEL for applications in X-ray spectrometry.

Other methods allow treating the response matrix of the system. For instance: the Singular Value Decomposition (SVD) (Golub and Van Loan, 1996), the Truncated Singular Value Decomposition (TSVD), the Modified Truncated Singular Value Decomposition (MTSVD) (Hansen et al., 1992), the Dump Singular Value Decomposition (DSVD) (Hansen et al., 1992), and the Tikhonov method (Hansen, 1999). 
In previous works, authors have applied TSVD and MTSVD methods jointly with regularization tools to unfold X-ray spectra using a Compton spectrometer in the radiodiagnostic energy range (Gallardo et al., 2006) and in the mammography energy range (Querol et al., 2011). However, the Compton spectrometer technique requires the using of detectors of high resolution, such as HPGe detectors (with liquid nitrogen cooling). This fact represents an important inconvenient to perform rapid and economical measurements. Gallardo et al. (2013) and Gallardo et al. (2015) tested the possibility of using flat panel detectors to obtain the primary X-ray spectrum. Flat panels are based on transforming the absorbed X-rays into charge carriers by means of a scintillator material that absorbs X-rays and converts them into visible light that passes onto a photodiode array. A semiconductor foil is used to convert visible light photons into charge carriers.

In this work, it has been developed a MCNP5 (X-5 Monte Carlo Team, 2003) model including the X-ray tube focus, a PMMA wedge attenuator and a flat panel. When the flat panel is irradiated, the absorbed dose in the scintillator layer (Gadolinium Oxysulfide, GOS) of the flat panel can be estimated using the F4MESH tally of MCNP5 at different positions. Absorbed dose in the GOS layer is obtained in the calculations considering the photon fluence and the mass energy-absorption coefficient $\left(\frac{\mu_{e n}}{\rho}\right)$ of GOS (Hubbell and Seltzer, 2011), provided by the National Institute of Standards and Technology (NIST). Several X-ray spectra have been considered varying the operation variables of the X-ray tube using the IPEM78 Catalogue (Cranley et al., 1997). With those spectra, the corresponding absorbed dose curves are obtained by simulation with a MCNP5 model. Once the dose curve corresponding to a determined X-ray spectrum is obtained, it can be estimated using unfolding techniques. The primary X-ray spectrum strongly depends on different parameters such as high voltage, filament current, high voltage ripple, anode angle and thickness of filter material. Slight variations of these parameters can produce non-negligible modifications in the Xray spectrum. It has been chosen the Tikhonov regularization method to unfold dose curves generated with MCNP.

The aim of the work is to provide information about both precision and accuracy of this unfolding method when estimating primary X-ray spectra using a flat panel and the Tikhonov regularization method. This information is relevant to weigh up the pros and cons of the scientific feasibility of the Tikhonov regularization to be used in quality control tests.

\section{Materials and Methods}

The Monte Carlo code MCNP5 (X-5 Monte Carlo Team, 2003) has been used to simulate the absorbed dose curve obtained when a flat panel with a PMMA wedge is irradiated. The model includes a point source, which simulates the X-ray focus, the PMMA wedge $(12.5 \times 12.5 \times 10 \mathrm{~cm})$ and the flat panel Hamamatsu C9312SK (Hamamatsu, 2007). The flat panel uses an indirect converting scintillator made of Gadolinium Oxysulfide (GOS), which is connected to the image sensor through a fiber optic layer. X-rays hit the 140 $\mu \mathrm{m}$ thick scintillator through the entrance window of $1 \mathrm{~mm}$ thick carbon. The $3 \mathrm{~mm}$ thick optic fiber is located behind the scintillator. The pixel size is $50 \times 50 \mu \mathrm{m}^{2}$. The distance between the X-ray focus and the flat panel is set to $85 \mathrm{~cm}$ and the radiation field to $15 \times 15 \mathrm{~cm}^{2}$. This flat panel is suitable to be used in the energy range between 20 and $110 \mathrm{keV}$ (Hamamatsu, 2007). Fig. 1 shows the geometry of the model including the X-ray focus (point source), the flat panel (with the main layers) and the wedge.

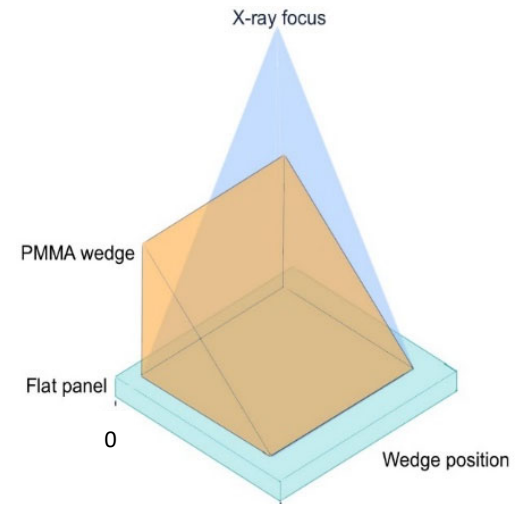

25

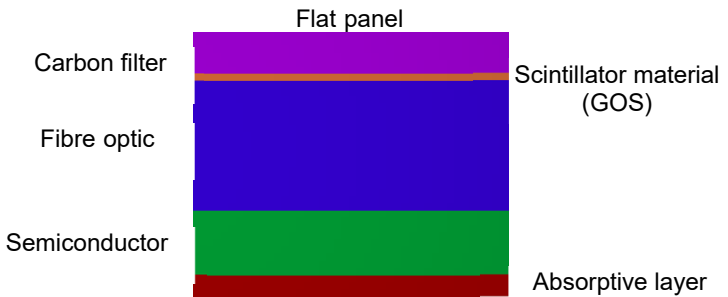


Fig. 1. Geometry model and flat panel layers.

Different design and working conditions of the tube have been considered (voltage, anode angle, high voltage ripple and aluminium filter thickness), as it can be seen in Table 1 . The corresponding spectrum for each set of conditions has been extracted from the IPEM78 Catalogue Report (Cranley et al, 1997) and used to characterize the X-ray source in the MCNP model. In this catalogue, the unattenuated photon spectra are given for tungsten targets, tube potential from $30 \mathrm{kV}$ to $150 \mathrm{kV}$, anode angles from $6^{\circ}$ to $22^{\circ}$, different anode materials and extrinsic filters. The ripple value can be varied from 0 to $30 \%$.

Table 1. Parameters of the X-ray spectra.

\begin{tabular}{lccc}
\hline & Minimum & Maximum & Increment \\
\hline Voltage $(\mathrm{kV})$ & 40 & 100 & 1 \\
Tungsten anode angle & $12^{\circ}$ & $22^{\circ}$ & $1^{\mathrm{o}}$ \\
High voltage ripple $(\%)$ & $0 \%$ & $30 \%$ & $5 \%$ \\
Aluminium filter thickness $(\mathrm{mm})$ & 1.5 & 3.5 & 0.5 \\
\hline
\end{tabular}

F4MESH tally $\left(1 / \mathrm{cm}^{2}\right)$ has been used to estimate the photon fluence in the GOS layer. The absorbed dose is calculated using the fluence-to-dose conversion factors for GOS (Hubbell and Seltzer, 2011). Result is a matrix of doses (120x120 elements) in $\mu \mathrm{Gy}$ per history. Collapsing the central rows of the matrix, an absorbed dose curve is obtained. It has been simulated 100 million photons to obtain relative errors lower than $2 \%$ in each cell mesh. MODE P, E has been activated to follow tracks of photons and electrons. A cutoff of $5 \mathrm{keV}$ for electrons has been used to reduce computation time. For each working condition, a dose curve per history is obtained. Fig. 2 shows the absorbed dose curves (in GOS) obtained when different parameters are varied.

Slight variations of these parameters produce weak modifications in the absorbed dose curves, as it can be seen in Fig. 2. The variation of the voltage produces changes in the whole dose curve, i.e. in all the positions of the flat panel (Fig. 2 (a)). It is possible to distinguish the absorbed dose curves produced by different spectra with an increment of $1 \mathrm{kV}$ between them. Furthermore, the difference in the dose curves is practically independent on the position in the wedge.

Regarding the variation of the anode angle, this effect is not so evident in the absorbed dose curve. In Fig. 2 (b) 3 groups of curves are plotted, each group with 10 different dose curves corresponding to 10 anode angles. As it can be seen, the variations in dose curve are very slight when the anode angle is modified. The effect is also observed in all the positions of the flat panel and it does not depend on the voltage considered. In Section 3 it is discussed the minimum variation in the anode angle required to produce a significant change in the unfolded spectrum.

Varying the voltage ripple (between 0 and 30\%), the voltage is modified following a synodal function, being modified all the energy spectrum. As it can be seen in Fig. 2 (c), an increment of 5\% of ripple produces different absorbed dose curves. The effect of the ripple is more evident in the widest part of the wedge (higher attenuation), being almost negligible in the thinnest extreme of the wedge. This effect seems to be independent of the voltage.

When the extrinsic filter is changed (increasing the aluminium thickness), the spectrum becomes harder, being cut in the lower energy range. This parameter produces the highest effect on dose curves. In this case (Fig. 2 (d)), the effect is also relevant in the widest side of the wedge, being negligible in the thinnest extreme. As it can be seen, an increment of $0.5 \mathrm{~mm}$ aluminium produces spectra that can be distinguished between them. 


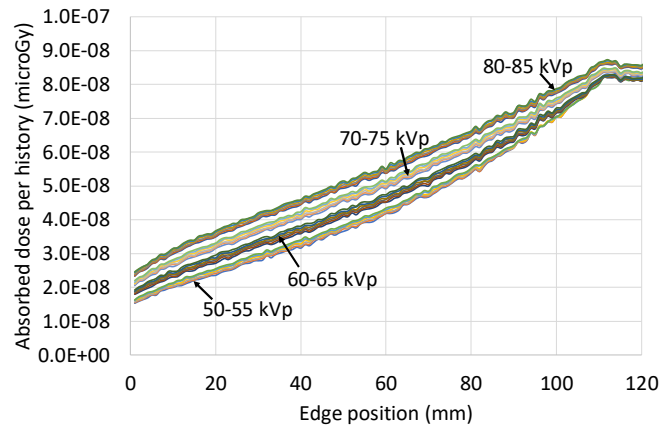

a) Voltage

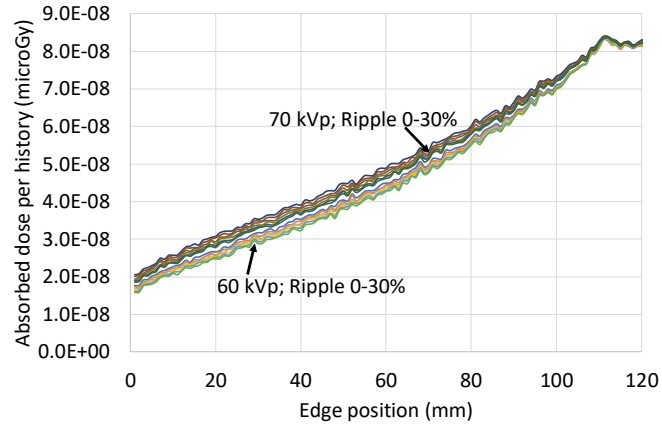

c) Voltage ripple

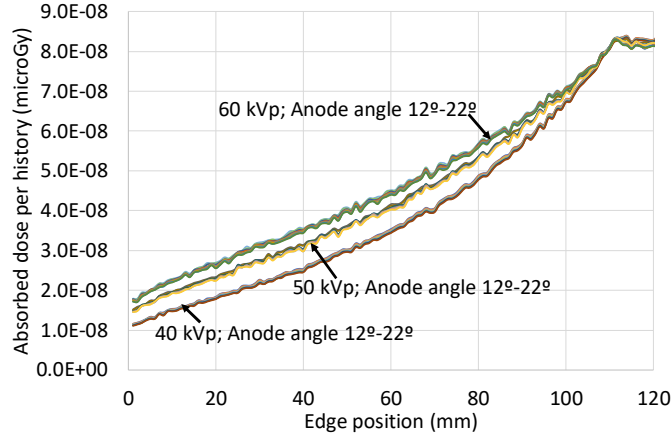

b) Anode angle

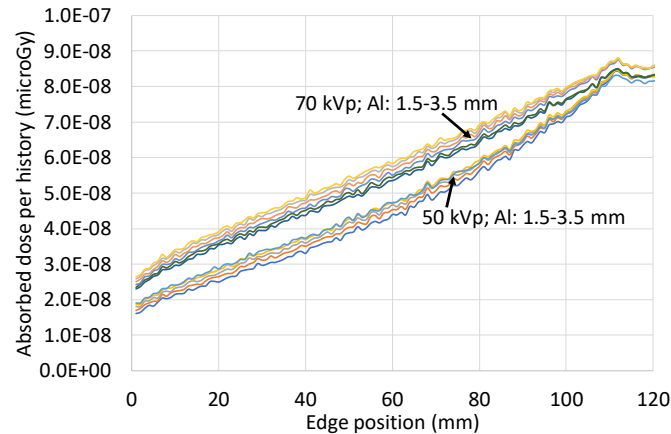

d) Aluminium filter thickness

Fig. 2. Simulated absorbed dose curves for different parameters.

The relation between the absorbed dose curve and the primary spectrum can be defined by a Response matrix (R), which can be obtained simulating the response of monochromatic photon beams:

where

$$
R \vec{S}=\vec{m}
$$

$\vec{S}$ is the unknown primary spectrum and

$\vec{m}$ is the absorbed dose curve registered.

The MCNP model can be used to determine the response matrix of the system. Thus, 135 monoenergetic spectra have been simulated covering the energy range between 15 and $150 \mathrm{keV}$, using $1 \mathrm{keV}$ energy bin. A dose curve is calculated for each monochromatic spectrum. The relation between energy and dose is not linear. In fact, for energies below the GOS K-edge $(50 \mathrm{keV})$, the maximum dose per emitted photon is found at $30 \mathrm{keV}$. Fig. 3 shows the response matrix (energy - absorbed dose) of the system. 


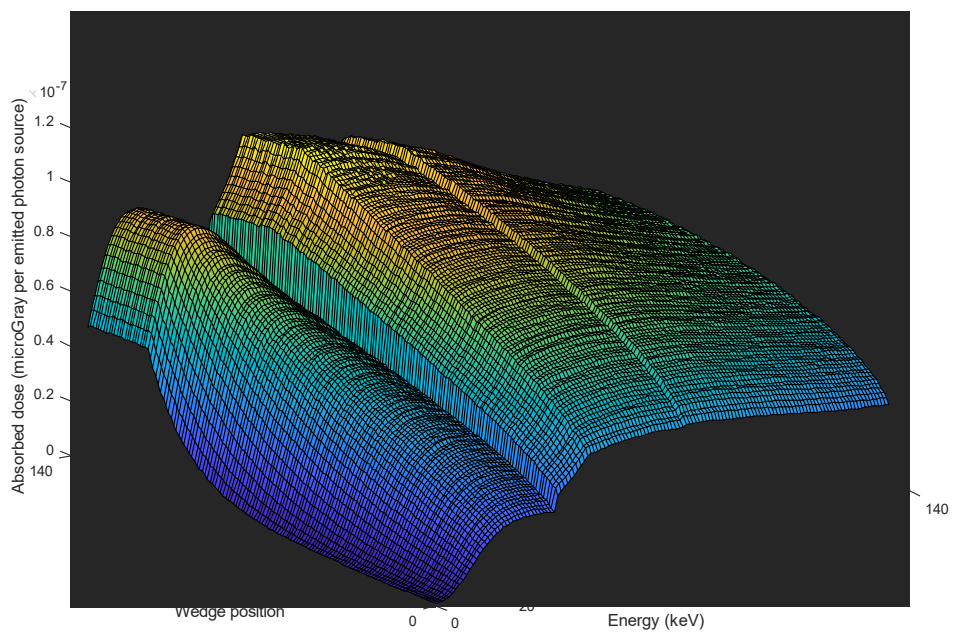

Fig. 3. Response matrix of the system.

When a dose curve is measured, and the response matrix is known, the primary X-ray spectrum can be estimated using unfolding techniques. In a previous work (Gallardo et al, 2015), authors used the Singular Value Decomposition (MTSVD) technique to unfold X-ray spectra when different scintillator are considered in a flat panel. Once $\mathrm{R}$ is known, the equation (1) theoretically permits to obtain the primary spectrum $\vec{s}$. However, this is an ill-posed problem, as R has a high condition number $\left(>10^{5}\right)$ being necessary to use regularization methods to inverse the matrix. Tikhonov regularization method (Hansen, 1999) is based on a modification of the Fredholm integral equation of the first kind that is intended to stabilize its solution:

where

$$
\mathrm{f}(\mathrm{x})=\|R \vec{s}-\vec{m}\|^{2}+\lambda\|L \vec{s}\|_{2}
$$

$\vec{S}$ is the unknown primary spectrum,

$\vec{m}$ is the absorbed dose curve registered,

$\mathrm{R}$ is the Response matrix,

The objective is to define the regularized solution $\tilde{\vec{s}}$ as the minimizer of the weighted residual norm and the side constraint:

$$
\tilde{\vec{s}}=\operatorname{argmin}\left\{\|R \vec{s}-\vec{m}\|_{2}^{2}+\lambda^{2}\|L \vec{s}\|_{2}^{2}\right\}
$$

where the regularization parameter $\lambda$ controls the weight given to minimization of the side constraint relative to minimization of the residual norm. L takes different forms in accordance with the order of regularization. The quality of the unfolding method results depends on the regularization parameter $(\lambda)$. For this reason, a reliable method for determination of the regularization parameter is essential to solve the inverse problem. One of the most extended method is the L-curve criterion (Hansen and Oleary, 1993). Other method for choosing an optimal regularization parameter is the Discrete Picard Condition (DPC), recognized by Varah, 1979 and analysed by Hansen, 1990. 


\section{Results and discussion}

For different working conditions (Table 1), the Tikhonov regularization method has been applied using the response matrix and dose curves. The parameter lambda has been set to 2.1 E-5 to accomplish with the Lcurve criterion.

Fig. 4 shows a comparison between theoretical spectra extracted from the IPEM78 Catalogue Report and unfolded spectra. All the spectra have been normalized to the area (arbitrary units in Y axis). To improve the understanding of this figure, it has only been represented the spectra corresponding to $50 \mathrm{kVp}$ and 70 $\mathrm{kVp}$, maintaining constant the anode angle $\left(12^{\circ}\right)$, the high voltage ripple $(0 \%)$ and the aluminium filter thickness $(1.5 \mathrm{~mm})$. In any case, this comparison is a representative example of all the cases analysed. It has been observed that there is a shift (2-3 keV) to lower energies of the unfolded spectrum (Bremsstrahlung continuous) respect to the theoretical one. In the case of voltages greater than $70 \mathrm{kVp}$, characteristic lines of tungsten appear in the theoretical spectra. These characteristic lines are lost during the unfolding process. The lack of characteristic lines in the unfolded spectra is explained by the loss of information when the pseudo inverse matrix is obtained. To quantitatively compare both theoretical and unfolded spectra, the Normalized Root Mean Square Error (NRMSE) has been calculated for each case. For $50 \mathrm{kVp}$ and $70 \mathrm{kVp}$, the NRMSE is $3 \%$ and $5 \%$, respectively. It has been analysed the high voltage range between 50 and 80 $\mathrm{kVp}$, obtaining a maximum NRMSE (IPEM - unfolding) lower than $10 \%$.

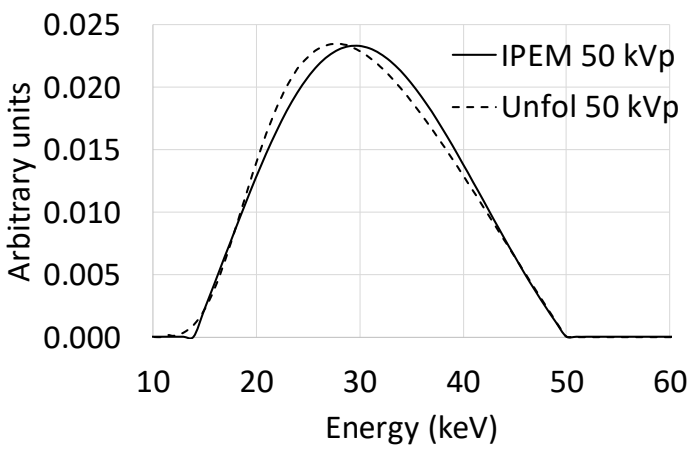

a) $50 \mathrm{kVp}$.

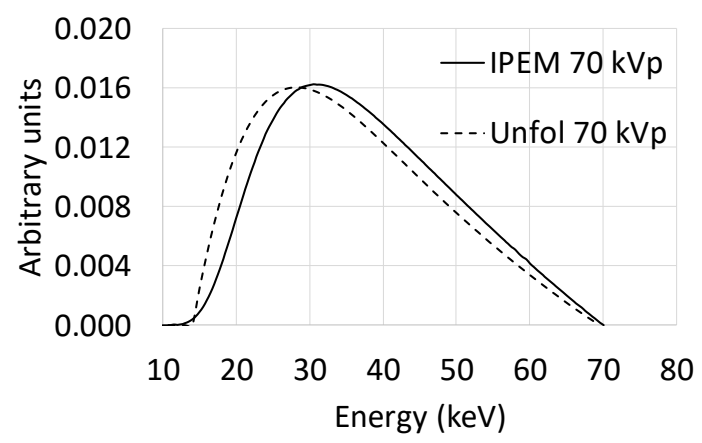

b) $70 \mathrm{kVp}$.

Fig. 4. Unfolded and theoretical spectra for 50 and $70 \mathrm{kVp}$.

To determine the accuracy of the Tikhonov regularization method in this specific frame, several dose curves have been simulated when one parameter of Table 1 is slightly varied.

Fig. 5 (a) shows the unfolded spectra of 51 and $55 \mathrm{kVp}$ and Fig. 5 (b) the corresponding to 70 and $75 \mathrm{kVp}$. In these cases, the anode angle $\left(12^{\circ}\right)$, the voltage ripple $(0 \%)$ and the aluminium filter thickness $(1.5 \mathrm{~mm})$ are maintained constant. With this regularization method it is possible to distinguish unfolded spectra when the voltage is varied with increments of $1 \mathrm{kV}$. In the voltage range between 50 and $60 \mathrm{kVp}$, the NRMSE obtained when the voltage is increased $1 \mathrm{kV}$ is $1.5 \%$ approximately. This value is greater (about $3.5 \%$ ) in the voltage range between 60 and $80 \mathrm{kVp}$ when increments of $1 \mathrm{kV}$ are also considered. For voltages between 50 and $60 \mathrm{kVp}$, it is achieved an acceptable agreement with the theoretical spectra. For higher voltages $(60-80 \mathrm{kVp})$, the agreement is also acceptable, but it is observed is some cases a disarrangement of about 1-3 $\mathrm{kVp}$ respect of the IPEM78 spectra. In all the cases analysed, a shifted to lower energies is observed in the Bremsstrahlung of the spectrum.

In Fig. 5 (c) the voltage $(60 \mathrm{kVp})$, the ripple $(0 \%)$ and the aluminium filter thickness $(1.5 \mathrm{~mm})$ are fixed, obtaining spectra for two different anode angles $\left(15^{\circ}\right.$ and $\left.20^{\circ}\right)$. It can be observed that both unfolded spectra present evident differences in the energy range between 15 and $40 \mathrm{keV}$, increasing the fluence of X-rays when the anode angle is increased. The NRMSE obtained when both spectra are compared is about $5 \%$. If the anode angle is changed $1^{\circ}$, the NRMSE is lower than $1 \%$. To ensure the differentiation between unfolded spectra it is recommended at increment of $2^{\circ}$ at least. 
In Fig. 5 (d) the high voltage ripple is varied (being the voltage fixed at $60 \mathrm{kVp}$ and the anode angle $12^{\circ}$ ). Theoretically, when the voltage ripple is increased (0-30\%), the fluence of X-rays in the higher energy range of the spectrum increases. This effect also appears in the unfolded spectra, but with lower intensity. When the ripple is varied with increments of 5\%, the NRMSE calculated when both spectra are compared is about $2.5 \%$. For unfolded spectra corresponding to voltage ripples of 0 and $30 \%$, the NRMSE is near $15 \%$.

Fig. 5 (e) shows the spectra obtained when the aluminium filter thickness is changed. It has been stated that increments of $0.5 \mathrm{~mm}$ of aluminium produces NRMSE of about $12 \%$. If the filter thickness is varied 0.1 $\mathrm{mm}$, the NRMSE obtained is $2 \%$, approximately.

In summary, the regularization method can identify variations of about $1 \mathrm{kVp}$ in voltage, $2^{\circ}$ in the anode angle, $5 \%$ in high voltage ripple and 0.1 aluminium filter thickness.

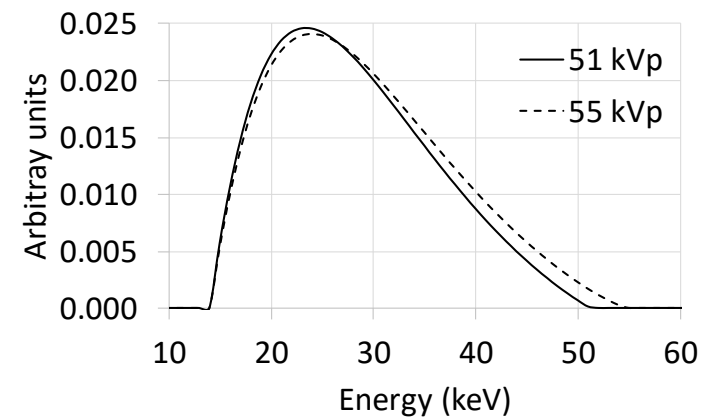

a) $51-55 \mathrm{kVp}, 12^{\circ}, 0 \%, 1.5 \mathrm{~mm} \mathrm{Al}$.

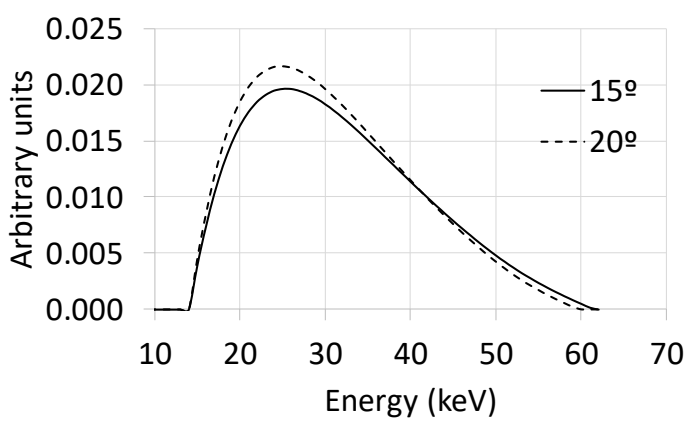

c) $60 \mathrm{kVp}, 15^{\circ}-20^{\circ}, 0 \%, 1.5 \mathrm{~mm} \mathrm{Al}$.

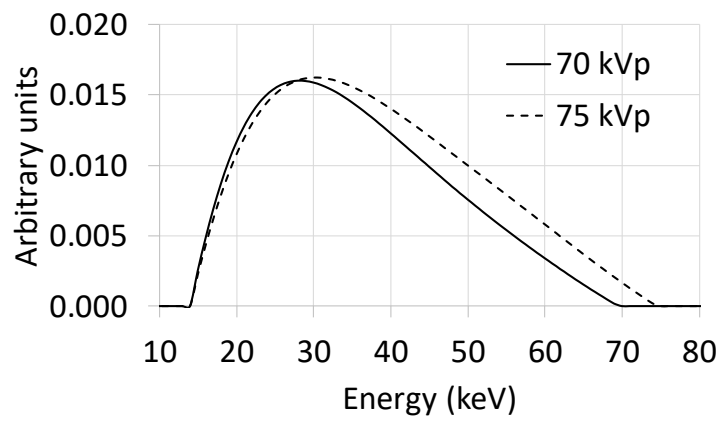

b) $70-75 \mathrm{kVp}, 12^{\circ}, 0 \%, 1.5 \mathrm{~mm} \mathrm{Al}$.

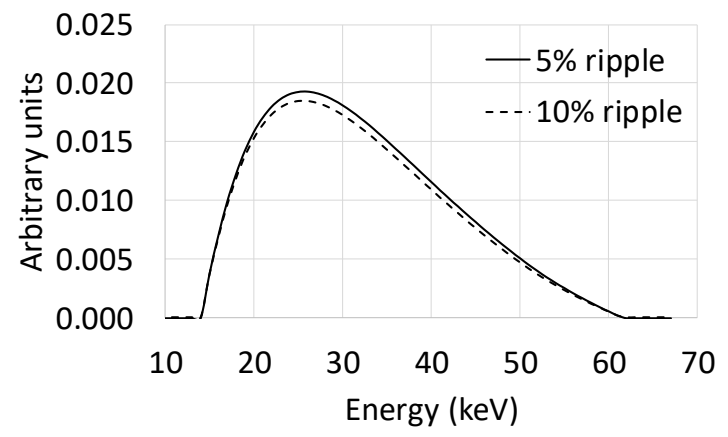

d) $60 \mathrm{kVp}, 12^{\circ}, 5 \%-10 \%, 1.5 \mathrm{~mm} \mathrm{Al}$.

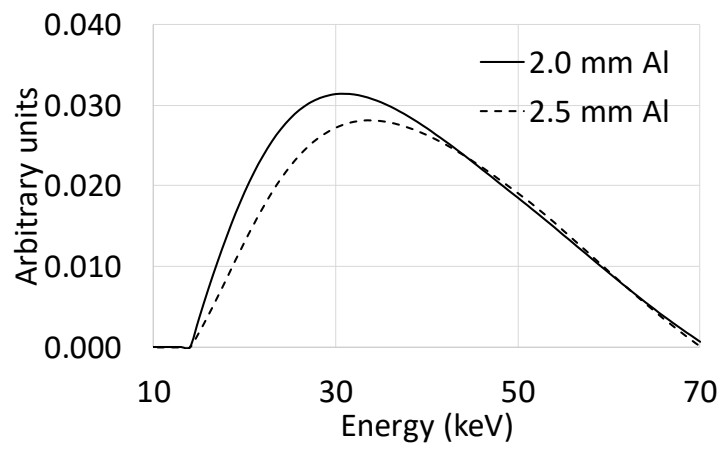

Fig. 5. Unfolded spectra for different conditions.

To quantitatively compare theoretical and unfolded spectra, mean energy, first and second Half Value Layers (HVL) have been calculated in every case. Normally, the HVL is experimentally obtained by overlapping aluminium or copper foils of certain thickness and certified purity between the X-ray focus 
and an ionization chamber. HVL for air kerma is calculated for X-ray according to the following expression (Thilander Klang, 1997):

$$
\frac{1}{2}=\frac{\sum_{i}\left(\frac{\mu_{e n}}{\rho}\right)_{\text {air }} N_{i} h v_{i} \exp \left(-\mu_{A l} H V L\right) \exp \left(-\mu_{A i r} d\right)}{\sum_{i}\left(\frac{\mu_{e n}}{\rho}\right)_{\text {air }} N_{i} h v_{i} \exp \left(-\mu_{A i r} d\right)},
$$

where $\left(\frac{\mu_{e n}}{\rho}\right)_{\text {air }}$ is the mass energy absorption coefficient for air (Hubbell and Seltzer, 2011); $\mu_{A i r}$ is the linear attenuation coefficient in air (Hubbell and Seltzer, 2011); $d$ is the distance between the X-ray focus and the ionization chamber; hvi is the photon energy of the $\mathrm{i}^{\text {th }}$ interval; $\mathrm{Ni}$ is the number of photons in the $\mathrm{i}^{\text {th }}$ energy interval and $\mu_{A l}$ is the linear attenuation coefficient in aluminium (Hubbell and Seltzer, 2011).

Table 2 summarizes the quality parameters (mean energy and the first and second HVL) of the unfolded spectra shown in Figure 5. The values corresponding to the theoretical spectra are included into brackets. The unfolding method underpredict the mean energy and the first and second HVL in any case. When the voltage is varied, it can be stated that the absolute maximum error of the mean energy is lower than $3 \mathrm{keV}$ in any of the cases considered, that means a relative maximum error lower than $7 \%$. Regarding the first and second HVLs, the maximum relative error between the theoretical and the unfolded spectra is about $15 \%$ in the whole voltage range analysed. It must be noticed that the best results are achieved for low voltage range $(50-55 \mathrm{kVp})$, with maximum relative errors of $2 \%, 1.5 \%$ and $1.6 \%$ for mean energy, first and second HVL, respectively.

When the anode angle is increased, the Bremsstrahlung continuous is shifted to lower energies, reducing the mean energy and softening the spectrum (lower HVLs). In the IPEM78 spectra $(60 \mathrm{kVp})$, when the anode angle is varied from $15^{\circ}$ to $20^{\circ}$, the mean energy is reduced $0.49 \mathrm{keV}(1.4 \%$ of relative variation $)$, the first HVL is reduced $0.9 \mathrm{~mm}(6.2 \%)$ and the second HVL $2 \mathrm{~mm}(5.6 \%)$. In the case of the unfolded spectra is important to highlight that all the cases are underestimated respect to the IPEM spectra. The maximum relative error between the unfolded and theoretical spectra is $6.0 \%$ for the mean energy and $13 \%$ for the HVLs.

The increasing of the ripple voltage produces also a softening of the spectrum and consequently, a reduction in the mean energy and the HVLs. In the theoretical spectra $(60 \mathrm{kVp})$, an increment of $5 \%$ in the voltage ripple produces a reduction of $0.5 \mathrm{keV}$ in the mean energy, and a reduction of $0.04 \mathrm{~mm}$ and $0.15 \mathrm{~mm}$ in the first and second HVLs, respectively. Comparing the unfolded and the theoretical spectra, the maximum relative error is $6.9 \%$ in the mean energy and $15 \%$ in the HVLs.

Finally, the effect of adding an aluminium filter is to harden the spectrum. The mean energy of the theoretical spectrum $(60 \mathrm{kVp})$ increases $0.9 \mathrm{keV}(2.5 \%)$ when the additional filter is increased in $0.5 \mathrm{~mm}$. In the unfolded spectra, the hardening is also predicted. The maximum relative error between the theoretical and the unfolded spectra is about $12 \%$. Regarding the HVL, the maximum relative error between the theoretical and unfolded spectra is about $15 \%$

Table 2. Mean energy, first and second HVL of unfolded spectra. Theoretical spectra values into brackets.

\begin{tabular}{|c|c|c|c|c|}
\hline Parameter & Description & $\begin{array}{c}\text { Mean Energy } \\
(\mathrm{keV})\end{array}$ & $\begin{array}{l}1^{\text {st }} \text { HVL } \\
\text { (mm Al) }\end{array}$ & $\begin{array}{l}2^{\text {nd }} \text { HVL } \\
\text { (mm Al) }\end{array}$ \\
\hline \multirow[t]{4}{*}{ Voltage } & $51 \mathrm{kVp} .12^{\circ}, 0 \%, 1.5 \mathrm{~mm} \mathrm{Al}$ & $31.1(31.4)$ & $1.28(1.30)$ & $3.07(3.10)$ \\
\hline & $55 \mathrm{kVp} .12^{\circ}, 0 \%, 1.5 \mathrm{~mm} \mathrm{Al}$ & $32.2(32.8)$ & $1.34(1.39)$ & $3.12(3.38)$ \\
\hline & 70 kVp. $12^{\circ}, 0 \%, 1.5 \mathrm{~mm} \mathrm{Al}$. & $35.6(38.1)$ & $1.50(1.75)$ & $3.90(4.44)$ \\
\hline & 75 kVp. $12^{\circ}, 0 \%, 1.5 \mathrm{~mm} \mathrm{Al}$ & $37.0(39.9)$ & $1.60(1.90)$ & $4.10(4.88)$ \\
\hline \multirow[t]{2}{*}{ Anode angle } & $60 \mathrm{kVp} . \mathbf{1 5}^{\circ}, 0 \%, 1.5 \mathrm{~mm} \mathrm{Al}$. & $32.5(34.2)$ & $1.30(1.44)$ & $3.10(3.55)$ \\
\hline & $60 \mathrm{kVp} . \mathbf{2 0}^{\circ}, 0 \%, 1.5 \mathrm{~mm} \mathrm{Al}$ & $31.7(33.7)$ & $1.22(1.35)$ & $3.00(3.35)$ \\
\hline \multirow[t]{2}{*}{ Voltage ripple } & $60 \mathrm{kVp} .12^{\circ}, \mathbf{5 \%}, 1.5 \mathrm{~mm} \mathrm{Al}$ & $32.1(34.5)$ & $1.29(1.48)$ & $3.11(3.65)$ \\
\hline & $60 \mathrm{kVp} .12^{\circ}, \mathbf{1 0} \%, 1.5 \mathrm{~mm} \mathrm{Al}$ & $31.6(33.6)$ & $1.28(1.44)$ & $3.10(3.50)$ \\
\hline \multirow[t]{2}{*}{ Aluminium filter } & $60 \mathrm{kVp} .12^{\circ}, 0 \%, 2.0 \mathrm{~mm} \mathrm{Al}$ & $31.4(35.7)$ & $1.51(1.75)$ & $3.72(4.25)$ \\
\hline & $60 \mathrm{kVp} .12^{\circ}, 0 \%, 2.5 \mathrm{~mm}$ Al. & $32.6(36.6)$ & $1.72(2.00)$ & $4.05(4.70)$ \\
\hline
\end{tabular}


From results shown in Figure 5 and Table 2, it can be stated that the precision of the method is acceptable, because slight variations in the operational parameters are detected and processed during the unfolding (variations of $1 \mathrm{kV}, 2^{\circ}$ anode angle, $5 \%$ ripple and $0.1 \mathrm{~mm} \mathrm{Al}$ ). However, the accuracy is not so good when comparing some quality parameters such as the mean energy, and the first and second HVL with those obtained from the theoretical spectra. In the energy range studied $(50-80 \mathrm{keV})$, maximum relative errors of $15 \%$ are found. This fact represents an inconvenient. However, the whole methodology is fast to implement providing a first approximation to the estimation of the primary spectra.

\section{Conclusions}

Unfolding of primary X-ray spectra in the radiodiagnostic energy range has been analysed by using the Monte Carlo code MCNP5. A Monte Carlo model including an X-ray source, a PMMA wedge and a flat panel has been developed to obtain dose curves from irradiation of the flat panel.

Simulation of different dose curves varying operational parameters of the X-ray tube has been done to perform a sensitivity analysis. Results show that slight changes in the primary spectrum produces weak variations in dose curves. The response matrix of the system has been obtained from several dose curves and the primary spectrum has been estimated. Direct unfolding is not possible, and a pseudo-inverse matrix has been obtained applying the Tikhonov regularization method. Results show that unfolding is possible, but some information is lost during the process. In general, it is produced a shift of the Bremsstrahlung continuous to lower energies and the characteristic X-ray lines of tungsten are lost.

It has been stated that the precision of the method is acceptable, because slight variations in the operational parameters are detected and processed during the unfolding, but the accuracy is not completely satisfactory when comparing some quality parameters with those obtained from theoretical spectra (15\% of maximum relative error). Despite this negative effect it is a low-cost technique to unfold X-ray spectra knowing a dose curve obtained with a flat panel. It does not require the using of HPGe detectors either nitrogen cooling systems. It is quite robust respect to the relative position between the source and the detector and allows obtaining fast estimations of the primary X-ray spectrum when the response function is calculated.

\section{References}

Cranley, K., Gilmore, B.J., Fogarty, G.W.A., Deponds, L., 1997. Catalogue of Diagnostic X-ray Spectra and Other Data, IPEM Report No. 78. The Institute of Physics and Engineering in Medicine, York.

Fernandez, J.E., Scot, V., Di Giulio, E., 2014. Spectrum unfolding in X-ray spectrometry using the maximum entropy method, Radiation Physics and Chemistry 95,154-157

Gallardo, S., Ginestar, D., Verdu, G., Rodenas, J., Puchades, V., Villaescusa, JI., 2006. X-ray spectrum unfolding using a regularized truncated SVD method, X-RAY SPECTROMETRY, 35(1), 63-70.

Gallardo S., Juste B., Pozuelo F., Querol A., Ródenas J. and Verdú G., 2013. Unfolding X-ray spectra using a flat panel detector, 35th Annual International Conference of the IEEE-Engineering-in-Medicine-andBiology-Society (EMBC), Osaka, Japan.

Gallardo S., Pozuelo F., Querol A., Ródenas J. and Verdú G., 2015. Application of the Monte Carlo method to analyze materials used in flat panel detectors to obtain X-ray spectra, Annals of Nuclear Energy 82, 240251.

Golub GH, Van Loan CF. Matrix Computations. Johns Hopkins University Press: Baltimore, MD, 1996.

Hamamatsu comercial catalogue, 2007. www.hamamatsu.com.

Hansen, P.C., 1990. The Discrete Picard Condition for discrete Ill-posed problems. BIT 30, 658-672.

Hansen, P.C., 1992. Analysis of discrete ill-posed problems by means of the L-curve. SIAM Rev. 34, 561580 . 
Hansen, P.C., Oleary, D.P., 1993. The use of the L-curve in the regularization of discrete ill-posed problems. SIAM J. Sci. Comput. 14, 1487-1503.

Hansen, P.C., 1999. Regularization tools Version 3.0 for Matlab 5.2. Numer. Algorithms 20, 195-196.

Hubbell, J.H., Seltzer, S.M., Last updated 2011. Tables of X-ray Mass Attenuation Coefficients and Mass Energy-Absorption Coefficients from $1 \mathrm{keV}$ to $20 \mathrm{MeV}$ for Elements $\mathrm{Z}=1$ to 92 and 48 Additional Substances of Dosimetric Interest. National Institute of Standards and Technology. www.nist.gov.

Los Arcos, J.M., 1996. Gamma-ray spectra deconvolution by maximum-entropy methods. Nucl. Instrum. Methods Phys. Res. Sec. A 369 (2-3), 634-636.

Ortiz-Rodriguez, JM., Martinez-Blanco, MR., Vega-Carrillo, HR., Diaz, EG., Fillol, AL., Villafane, RM., Merino, JML., Araque, JEG., 2011. Performance of artificial neural networks and genetical evolved artificial neural networks unfolding techniques, Revista Mexicana de Física, 57(1), 89-92.

Querol, A., Gallardo, S., Rodenas, J., Verdú, G., 2011. Application of the MTSVD and Tikhonov unfolding methods for reconstruction of primary x-ray spectra of mammography equipment with silicon detector, Nuclear Technology 175(1), 63-72.

Reginatto, M., Goldhagen, P., Neumann, S., 2002. Spectrum unfolding, sensitivity analysis and propagation of uncertainties with the maximum entropy deconvolution code MAXED. Nucl. Instrum. Methods Phys. Res. Sec. A 476, 242-246.

Reginatto, M., 2003. The "multi-channel" unfolding programs in the UMG package: MXD_MC33GRV_MC33 and IQU_MC33, UMG package version 3.3, Physikalisch Technische Bundesanstalt (PTB).

Reginatto, M., Zimbal, A., 2008. Bayesian and maximum entropy methods for fusion diagnostic measurements with compact neutron spectrometers. Rev. Sci. Instrum., 79.

Thilander Klang, A., 1997. Diagnostic quality and absorbed dose in mammography. Influence of X-ray spectra and breast anatomy, PhD Thesis, Department of Radiation Physics, University of Göteborg, Sweden.

X-5 MONTE CARLO TEAM, 2003. MCNP - A General Monte Carlo N particle Transport Code, Version 5 LA-UR-03-1987. Los Alamos National Laboratory.

Varah, J.M., 1979. A practical examination of some numerical methods for linear discrete Ill-posed problems. SIAM Rev. 21, 100-111. 